\title{
Ear discharge in children presenting with acute otitis media: observational study from UK general practice
}

Lindsay Smith, Paul Ewings, Caroline Smith, Matthew Thompson, Anthony Harnden and David Mant

\section{ABSTRACT \\ Background \\ National Institute for Health and Clinical Excellence (NICE) guidance to treat otitis media in older children immediately with antibiotics only if they have ear discharge is based on limited evidence.}

Aim

To determine the clinical significance and outcome of ear discharge in children with acute otitis media, in routine clinical practice.

\section{Design of study}

Observational cohort study of children with acute otitis media comparing those with and without ear discharge at presentation.

\section{Setting}

Primary care in East Somerset.

\section{Method}

Two hundred and fifty-six children aged 6 months to 10 years were recruited from primary care. Clinical features and other characteristics were recorded at presentation. Follow-up was undertaken at 2 weeks and 3 months.

\section{Results}

Children with otitis media who present with ear discharge are much more likely to be treated with antibiotics irrespective of age (adjusted odds ratio 15 $95 \%$ confidence interval $[\mathrm{Cl}]=3$ to 66$)$. Most with discharge have proven bacterial infection (58\%, 95\% $\mathrm{Cl}=42$ to $72 \%)$. They have a more severe systemic illness, with higher axillary temperature (80\% increase in odds of ear discharge for each additional degree centigrade, $P=0.02)$, pulse rate (9\% increase in odds for each extra beat, $P<0.001$ ), and Yale score (mean 10.5 versus $9.0, P=0.003$ ). They may also have an increased likelihood of adverse outcome (adjusted odds ratio of pain at 1 week 2.9; further episodes of acute otitis media 3.3; hearing difficulty at 3 months 4.7; all $P<0.10$ ).

\section{Conclusion}

Ear discharge defines a group of children with otitis media who are sicker and may be at higher risk of adverse outcome. NICE guidance to treat them with antibiotics is supported.

\section{Keywords}

acute; general practice; family practice; otorrhea; otitis media.

\section{INTRODUCTION}

The National Institute for Health and Clinical Excellence (NICE) guidance on treatment of acute otitis media in children states that antibiotic prescribing is usually not necessary. ${ }^{1}$ The justification for this guidance is that most children with acute otitis media recover within a few days without antibiotics. While antibiotics may benefit some children, pain relief is not immediate, the average reduction in duration of pain is only a few hours, and there is no impact on long-term hearing or ear, nose, and throat (ENT) referrals. ${ }^{2-10}$ However, the NICE guidance does recommend that GPs should 'consider' immediate antibiotic treatment for two groups of children with acute otitis media - the very young (age <2 years) and those with ear discharge. The stated justification for the latter is that a posthoc meta-analysis of the clinical trial data suggests that only three children need to be treated for one less child to experience pain or fever at the 3-7-day assessment point. ${ }^{11}$

Subgroup analyses are not usually considered reliable evidence of effect to underpin national guidelines, as they generate hypotheses rather than

L Smith, MCLinSci, MD, FRCP, FRCGP, research lead, East Somerset Research Consortium, Westlake Surgery, West Coker, Somerset. P Ewings, BSc, MSc, PhD, CStat, director; C Smith RGN, RM, research associate, NIHR Research Design Service (South West), Taunton \& Somerset NHS Foundation Trust, Musgrove Park Hospital, Taunton. M Thompson, MPH, DPhil MRCGP; A Harnden, FRCGP, FRCPH; D Mant, FRCGP, FRCP, Oxford University Department of Primary Health Care, Oxford.

Address for correspondence

Dr Lindsay Smith, East Somerset Research Consortium, Westlake Surgery, West Coker, Somerset, BA22 9AH.

E-mail: research@esrec.nhs.uk

Submitted: 7 April 2009; Editor's response: 2 June 2009; final acceptance: 14 July 2009.

(C)British Journal of General Practice 2010; 60: 101-105.

DOI: 10.3399/bjgp10X483148 


\section{How this fits in}

The majority of children seen in primary care with ear discharge secondary to acute otitis media have a bacterial cause on a standard swab taken at presentation. They do appear to be a separate clinical group, perhaps representing the more extreme range of otitis media, and may suffer a greater number of longer-term problems. In this study most were given antibiotics by the GP at presentation, but this needed changing in a significant minority of children. Such antibiotic prescribing is in line with recent National Institute for Health and Clinical Excellence (NICE) guidance, but this study suggests that a routine ear swab may help guide future short-term management.

proving them. Moreover, ear discharge is simply an indication that the tympanic membrane has perforated; there is little evidence that presentation with ear discharge is associated with a different underlying pathology needing different treatment. One recent trial reports that spontaneous perforation of the tympanic membrane is associated with higher short-term treatment failure after 5 days of antibiotics, but the numbers were small (only eight children suffered spontaneous perforation) and there was no difference in children treated for 10 days. ${ }^{12} \mathrm{~A}$ paper from the US in 1993 reported that children aged $>1$ year presenting with pustular discharge had poorer long-term outcome at 2 months' follow-up, ${ }^{13}$ but the only other paper that can be identified to support this contention was derived from one of the clinical trials included in the above subgroup meta-analysis. ${ }^{14}$

This cohort study was conducted in UK general practice to confirm how frequently otitis media in children presents with ear discharge in routine clinical practice and whether it usually has a bacterial cause, to explore whether children with ear discharge are in any way different from those without discharge, and to assess whether disease severity and the frequency of adverse outcome is indeed higher in children with discharge.

\section{METHOD}

Children aged 6 months to 10 years presenting with acute otitis media (GP clinical diagnosis) were recruited from primary care in East Somerset during the three winters from 2003 to 2006. Recruitment took place during the normal working day from 21 general medical practices and from one out-ofhours primary care centre during evenings and weekends. The practices and out-of-hours GPs agreed in advance to participate, and were asked to refer any child with otitis media irrespective of severity. Informed consent was obtained by the research nurse, who visited the patient within 4 hours in order to obtain demographic and baseline clinical data and, when possible, to obtain a photograph of the tympanic membrane to confirm the diagnosis. All photographs were later reviewed by two experienced GPs including one of the authors. Swabs were obtained by either the referring GP or the research nurse, from the ear canal where discharge was visible to the GP or research nurse, or if the parent had noticed discharge within the previous 12 hours. Swabs were transported to the laboratory the same day, for routine bacterial culture.

The sociodemographic variables recorded at presentation were age, sex, parental age, parental smoking habit, history of breast-feeding, and postcode (from which the deprivation index was derived). The clinical history variables recorded were presenting symptoms, number of previous episodes of acute otitis media reported by the parents, parental global assessment of severity (on a 0-5 scale), and any other significant comorbidity. The clinical variables recorded were axillary temperature (AND model DT-502EC digital thermometer), pulse rate (counted at the wrist for 1 minute), nurse global clinical assessment (again on a $0-10$ scale), and the Yale score (a tool that has been used to objectively assess overall severity of illness in children presenting with acute infections in emergency department settings; it includes a scoring of six items: colour, hydration, response to greeting, state variation, respiratory effort, and activity level). ${ }^{15}$ Parents were given a symptom diary to record clinical symptoms for the 7 days following recruitment. The nurse visited or phoned the child's parents at 14 days, and again at 3 months, to collect the diary and ascertain further episodes of infection or antibiotic use; she also enquired whether the parents believed that the child had any residual hearing problems, but did not conduct a formal hearing test.

Follow-up was completed for all 38 children with ear discharge and $94 \%(205 / 218)$ of children without ear discharge. The initial study aim was to recruit 300 children with acute otitis media, of whom it was anticipated 60 would have ear discharge; as only 256 were actually recruited, the estimates of both the prevalence of ear discharge and its clinical consequences are less precise than originally planned. The main antecedent hypotheses were that ear discharge was likely to be associated with more antibiotic treatment by GPs, poorer clinical outcome at 1 week, and more hearing problems persisting at 3 months.

The main analysis compared children presenting with and without ear discharge. Estimates of prevalence are reported with 95\% confidence intervals (Cls). The associations between ear 
Table 1. Risk factors for ear discharge in children with acute otitis media.

\begin{tabular}{|c|c|c|c|c|}
\hline & $\begin{array}{l}\text { Ear discharge } \\
\quad(n=38)\end{array}$ & $\begin{array}{c}\text { No ear } \\
\text { discharge }(n=218)\end{array}$ & $\begin{array}{l}\text { Crude OR } \\
(95 \% \mathrm{Cl})\end{array}$ & $\begin{array}{l}\text { Adjusted OR }{ }^{a} \\
(95 \% \mathrm{Cl})\end{array}$ \\
\hline \multicolumn{5}{|l|}{ Demographic factors } \\
\hline Males, $n(\%)$ & $16(42)$ & $97(44)$ & 0.91 (0.45 to 1.82$)$ & - \\
\hline Age, median years (IQR) & $3(1-5)$ & $3(1-5)$ & 0.93 (0.81 to 1.06$)$ & - \\
\hline Parent(s) smoke, $n$ (\%) & $12(32)$ & $86(40)$ & 0.70 (0.34 to 1.47$)$ & - \\
\hline Ever breast-fed, $n$ (\%) & $25(66)$ & $147(67)$ & 0.93 (0.45 to 1.92$)$ & - \\
\hline Deprivation score, median (IQR) & $12.7(10.3-16.2)$ & 2) $12.7(9.2-18.4)$ & 0.99 (0.94 to 1.04$)$ & - \\
\hline \multicolumn{5}{|l|}{ Clinical history of acute otitis media } \\
\hline Any previous episodes, $n(\%)$ & $26(68)$ & $133(61)$ & 1.38 (0.66 to 2.89$)$ & - \\
\hline First episode age, median years (IQR) & $1(0-2)$ & $1(0.03-3)$ & $0.93(0.76$ to 1.14$)$ & - \\
\hline Number infections ever, median (IQR) & $2(0-6)$ & $1(0-4)$ & 1.04 (0.99 to 1.10$)$ & $1.10(1.03$ to 1.18$)$ \\
\hline \multicolumn{5}{|l|}{ Clinical presentation } \\
\hline Earache in both ears, $n(\%)$ & $4(11)$ & $120(56)$ & 0.09 (0.03 to 0.27$)$ & 0.03 (0.01 to 0.13$)$ \\
\hline Vomited, $n(\%)$ & $15(40)$ & $56(26)$ & $1.88(0.91$ to 3.84$)$ & $1.18(0.41$ to 3.45$)$ \\
\hline Axillary temperature, median ${ }^{\circ} \mathrm{C}(\mathrm{IQR})$ & $36.8(36.5-38.1)$ & 1) $36.5(36.0-37.1)$ & 1.76 (1.28 to 2.43$)$ & 1.80 (1.11 to 2.93$)$ \\
\hline Pain score ${ }^{b}$, median (IQR) & $5.3(2.5-7.2)$ & $5.6(3.0-7.2)$ & 0.96 (0.84 to 1.10$)$ & - \\
\hline Pulse rate, median (IQR) & $130(124-142)$ & $104(90-124)$ & 1.07 (1.04 to 1.09$)$ & 1.09 (1.05 to 1.12$)$ \\
\hline
\end{tabular}

$I Q R=$ interquartile range. $O R=$ odds ratio. ${ }^{a} \mathrm{O} R$ s from logistic regression; adjusted version adjusts for those variables with $P<0.1$ from unadjusted analyses. ${ }^{b}$ Pain score assessed by parent for 24 hours before presentation.

discharge and both presenting characteristics and clinical outcomes were estimated by calculating odds ratios using logistic regression; adjusted odds ratios were estimated from a model including all variables associated with ear discharge, at a statistical significance of $P<0.1$ from the unadjusted analyses.

\section{RESULTS}

\section{Prevalence and microbiology}

Of the 256 children recruited with acute otitis media, 38 (15\%, 95\% Cl = 11 to $20 \%)$ had ear discharge (otorrheoa) at first presentation. Children with ear discharge had the same median age ( 3 years, $P=$ $0.20)$. A recognised bacterial pathogen was identified from culture of the discharge in 22 children $(58 \%$, $95 \% \mathrm{Cl}=42$ to $72 \%$ ). The infecting bacteria were Streptococcus pneumococci $(n=5)$, Streptococcus A $(n=7)$, Staphylococcus aureus $(n=7)$,
Pseudomonas $(n=2)$ and Haemophilis influenzae $(n$ = 3); two children had mixed infections. Of these 24 pathogens, 21 were treated with antibiotics to which they were sensitive (16 initial and five changed when the swab result was known) and three antibiotics to which they were resistant that were not changed, as the child was better when contacted.

\section{Clinical presentation}

Table 1 shows the demographic and clinical characteristics associated with ear discharge. Neither age, sex, parental smoking, or social deprivation predicted whether a child would present with a discharging ear. The only aspect of the clinical history that predicted ear discharge was the number of previous episodes of acute otitis media, with a $10 \%$ increase in odds for each additional infection $(P$ $=0.007)$. Irrespective of the presence of ear discharge, most children with otitis media were

Table 2. Clinical consequences of ear discharge in children with acute otitis media.

\begin{tabular}{|c|c|c|c|c|}
\hline & $\begin{array}{c}\text { Ear discharge } \\
n / N(\%)\end{array}$ & $\begin{array}{c}\text { No ear discharge } \\
n / N(\%)\end{array}$ & $\begin{array}{l}\text { Crude OR } \\
(95 \% \mathrm{Cl})\end{array}$ & $\begin{array}{l}\text { Adjusted OR }{ }^{\mathrm{a}} \\
\quad(95 \% \mathrm{Cl})\end{array}$ \\
\hline GP prescribed antibiotic & $35 / 38(92)$ & $97 / 218(44)$ & 14.6 (4.3 to 48.8$)$ & 14.7 (3.3 to 65.6) \\
\hline Pain persisted at 1 week & $13 / 38(34)$ & $44 / 218(20)$ & 2.06 (0.97 to 4.34$)$ & $2.90(0.89$ to 9.46$)$ \\
\hline $\begin{array}{l}\text { Further episode of acute otitis media } \\
\text { within } 3 \text { months }\end{array}$ & $15 / 34(44)$ & 45/194 (23) & 2.61 (1.23 to 5.56$)$ & 3.34 (0.92 to 12.1$)$ \\
\hline $\begin{array}{l}\text { Parents reported residual hearing problem } \\
\text { at } 3 \text { months }\end{array}$ & $8 / 36(22)$ & 22/198 (11) & 2.29 (0.93 to 5.63$)$ & $4.67(0.80$ to 27.4$)$ \\
\hline
\end{tabular}


crying or irritable $(215,84 \%)$, and had night disturbance $(213,83 \%)$ and ear pain $(231,90 \%)$.

Ear discharge was more usually associated with unilateral pain $(89 \%$ versus $44 \%, P=0.001)$ but it made little difference to global assessment of severity, although parents recorded significantly higher mean scores than nurses (4.6 versus 1.7). However, children with discharge had objective signs of more severe illness, with a higher mean Yale score (10.5 versus $9.0, P=0.003$ ) and a tendency to higher axillary temperatures ( $80 \%$ increase in odds for each additional degree centigrade, $P=0.02$ ) and pulse rates $(9 \%$ increase in odds for each extra beat, $P<0.001)$.

\section{Clinical outcome and consequences}

Table 2 shows that the vast majority of children with ear discharge, unlike the children without discharge, were prescribed antibiotics by the GP (92\% versus $44 \%$; adjusted odds ratio $15,95 \% \mathrm{Cl}=3$ to 66 ). Thirty-two were prescribed amoxicillin, two erythromycin, one topical gentamicin, and three no antibiotics. It was therefore not possible to estimate the impact of antibiotic prescribing on outcome in children with discharge, although in most $(30 / 38$, $79 \%)$ the discharge had resolved within a week. Persistence of discharge was more likely in children in whom the bacterial swab had identified a bacterial pathogen (36\% versus $0 \%, P=0.01)$. Persistence of pain for more than 1 week was more likely in children with ear discharge on univariate analysis, but after adjustment for other variables the increased odds were not statistically significant (odds ratio $2.9, P=0.077$ ). Similarly, ear discharge was associated with increases in the likelihood of a further episode of acute otitis media within 3 months (odds ratio $3.3, P=0.066$ ) and of residual hearing problems (odds ratio $4.7, P=0.088$ ), which just failed to achieve statistical significance.

\section{DISCUSSION}

\section{Summary of main findings}

Ear discharge in children with acute otitis media is mostly bacterial in origin, and GPs almost always treat it with an antibiotic. In most children (4 out of 5) the discharge resolves within a week. Children with ear discharge are more likely to have had a previous episode of otitis media and they present with a more severe systemic illness - higher Yale scores, axillary temperatures, and pulse rates. They probably also have a higher likelihood of adverse outcomes prolonged pain, further episodes of acute otitis media, and parent-reported hearing problems. Despite the $P$-values being not significant at the conventional level, all the odds ratios are credible and consistent.

\section{Strengths and limitations of the study}

Important strengths of the study are that it was conducted in routine clinical practice, the diagnosis was validated by imaging the tympanic membrane, and follow-up was virtually complete. The two main weaknesses of the study are the limited number of cases of ear discharge identified (which leads to wide Cls on estimates of outcome), and the failure to recruit all children presenting with otitis media during the study period; consent was not obtained to review practice records for missed cases who were not referred to the research nurse. The expected annual consultation rate for otitis media in UK children is about $80 / 1000$. It was not possible to compare the present study population to this because not all the local general medical practices took part, although their patients were seen by the out-of-hours service locally. The age and sex of children in the study does not suggest substantial selection bias but the $15 \%$ prevalence of discharge observed in this study is higher than previously reported from the UK (10\%), Netherlands (14\%), and North America (9\%). ${ }^{16}$ It is also higher than suggested by the clinical experience of the authors. This may simply reflect local policy of discouraging attendance with uncomplicated earache but it could also reflect a tendency not to recruit mild cases of otitis media to the study. However, it is important to note that the situation is very different in less economically developed countries where most children with acute otitis media present with ear discharge (for example, 96\% in Sudan). ${ }^{17}$ This presumably reflects poor access to care, poor nutrition and immunity, and the chronic and untreated nature of many conditions presenting as acute problems.

\section{Comparison with existing literature}

Current knowledge of the clinical course of otitis media with discharge comes from the antibiotic treatment trials, particularly the subgroup metaanalysis of the individual patient data from these trials, ${ }^{11}$ cited in the introduction to this paper. However, a number of other trials have explored the effect of induced ear discharge (myringotomy) they showed worse 24-hour outcome but no difference in 7-day resolution or longer-term outcomes. ${ }^{18-20}$ The present finding that about $60 \%$ of cases have an identifiable bacterial cause is entirely consistent with previously published data, ${ }^{19}$ as is the range of bacteria identified, ${ }^{19-22}$ although it was not possible to find any other UK data subsequent to the most recent changes in vaccine policy. No studies suggest that bacterial and viral infection can be distinguished on the basis of clinical presentation without microbiology. ${ }^{21,22}$ Although one paper suggests that bacterial findings are poorly correlated 
with clinical course, this paper also reported that all cases of persistent discharge were attributable to antibiotic resistance of the bacterium identified. ${ }^{21}$

\section{Implications for future research and clinical practice}

The full explanatory text of the NICE guideline displays some lack of consensus about the recommendation to treat children with ear discharge differently. ${ }^{1}$ It states that the secondary data analysis on which the recommendation is made should be interpreted with caution for two reasons: the inherent unreliability of post-hoc subgroup analyses; and although the analysis suggests that only three children with otorrheoa need to be treated to prevent one additional child from having pain at days $3-7$, the pain at this point is mild and might be avoided with adequate analgesia. However, the present data strongly support the guideline recommendation by confirming that adverse outcome is more common in children with ear discharge, and providing an explanation of why antibiotics are likely to be more effective - rupture of the ear drum is clearly an indicator of a more severe, usually bacterial, infection. The present data also suggest that antibiotic resistance to amoxicillin is a problem affecting outcome and, arguably, a $20 \%$ nonresolution rate means that culture of pus from a discharging ear at first consultation to check antibiotic sensitivity should be standard practice because it would enable a prompt change of antibiotic if the infection is resistant or the cessation of antibiotic if the culture is negative. A final definitive answer on the cost-benefit of antibiotic treatment would require a further clinical trial limited to this subgroup of children. Such a trial may now not attract support from either doctors or parents.

\section{Funding body}

Children's Research Fund.

Ethical approval

Somerset REC (06/Q2202/70).

Competing interests

The authors have stated that there are none.

\section{Acknowledgements}

We would like to thank those children and parents who took part in the study; the GPs who referred patients to us; and the SW Ambulance Trust who permitted access through their out-of-hours service.

\section{Discuss this article}

Contribute and read comments about this article on the Discussion Forum: http://www.rcgp.org.uk/bjgp-discuss

\section{REFERENCES}

1. National Institute for Health and Clinical Excellence (NICE). Prescribing of antibiotics for self-limiting respiratory tract infections in adults and children in primary care. NICE Clinical Guideline 69. London: NICE, 2008. http://www.nice.org.uk/Guidance/CG69 (accessed 12 Aug 2009)

2. Glasziou PP, Del Mar CB, Saunders SL, Hayem M. Antibiotics for acute otitis media in children. Cochrane Database Syst Rev 2003; 4: CD000219.

3. Kontiokari T, Koivunen P, Neimela M, et al. Symptoms of acute otitis media. Pediatr Infect Dis J 1998; 17(8): 676-679.

4. Rosenfeld RM. Diagnostic certainty for acute otitis media. Int J Pediatr Otorhinolaryngol 2002; 64(2): 89-95.

5. Little P, Gould C, Williamson I, et al. Pragmatic randomized controlled trial of two prescribing strategies for childhood otitis media. BMJ 2001; 322(7282): 336-342.

6. Coco AS. Cost-effectiveness analysis of treatment options for acute otitis media. Ann Fam Med 2007; 5(1): 29-38.

7. Glasziou PP, Delmar CB, Sanders SL, Hayem M. Are antibiotics indicated as initial treatment for children with acute otitis media? A meta-analysis. BMJ 1997; 314(7093): 1526-1529.

8. Burke P, Bain J, Robinson D, Dunleavey J. Acute red ear in children: controlled trial of non-antibiotic treatment in general practice. $B M J$ 1991; 303(6802): 558-562.

9. Damoiseaux RA, van Balen FA, Hoes AW, et al. Primary care based randomized, double blind trial of amoxycillin versus placebo for acute otitis media in children aged under 2 years. BMJ 2000; 320(7231): 350-354.

10. Williamson I, Benge S, Mullee M, Little P. Consultations for middle ear disease, antibiotic prescribing and risk factors for reattendance: a case linked cohort study. Br J Gen Pract 2006; 56(524): 170-175.

11. Rovers MM, Glasziou P, Appelman CL, et al. Antibiotics for acute otitis media: a meta-analysis with individual patient data. Lancet 2006 368(9545): 1429-1435.

12. Hendrickse WA, Kusmiesz H, Shelton S, Nelson JD. Five vs. ten days of therapy for acute otitis media. Pediatr Infect Dis J 1988; 7(1): 14-23.

13. Froom J, Culpepper L, Bridges-Webb C, et al. Effect of patient characteristics and manifestations of on the outcome of acute otitis media at 2 months. Arch Fam Med 1993; 2(8): 841-846.

14. Little P, Gould C, Moore M, et al. Predictors of poor outcome and benefit from antibiotics in children with acute otitis media: pragmatic randomized trial. BMJ 2002; 325(7354): 22.

15. McCarthy PL, Lembo RM, Fink HD, et al. Observation, history, and physical examination in diagnosis of serious illnesses in febrile children less than or equal to 24 months. J Pediatr 1987; 110(1): 26-30.

16. Froom J, Culpepper J, Green L, et al. A cross-national study of acute otitis media: risk factors, severity, and treatment at initial visit. Report from the International Primary Care Network (IPCN) and the Ambulatory Sentinel Practice Network (ASPN). J Am Board Fam Practice 2001; 14(6): 406-417.

17. Hussain M, Ali E, Ahmed H. Otitis media in Sudanese children: presentation and bacteriology. East Afr Med J 1991; 68(9): 679-685

18. Van Buchem FL, Dunk JH, van’t Hof MA. Therapy of acute otitis media: myringotomy, antibiotics, or neither? A double-blind study in children. Lancet 1981; 2(8252): 883-887.

19. Van Buchem FL, Peeters MF, van't Hof MA. Acute otitis media: a new treatment strategy. Br Med J (Clin Res Ed) 1985; 290(6474): 1033-1037.

20. Engelhard D, Cohen D, Strauss N, et al. Randomised study of myringotomy, amoxycillin/clavulanate, or both for acute otitis media in infants. Lancet 1989; 2(8655): 141-143.

21. Palmu AA, Herva E, Savolainen $\mathrm{H}$, et al. Association of clinical signs and symptoms with bacterial findings in acute otitis media. Clin Infect Dis 2004; 38(2): 234-242.

22. Thompsen V, Olsen J, Sorensen H, Thompsen J. Bacteriology and antibiotics in acute suppurative otitis media. J Otolaryngol 1976; 5: 289-297. 